\title{
Zur Renzension angeboten
}

Amsler, Mark: Etymology and grammatical discourse in Late Antiquity and the Early Middle Ages. - 1989. VIII, 279 S.

Athapaskan linguistics. Current perspectives on a language family. Ed. by Eung Do Cook and Keren Rice. - 1989. VIII, 645 S.

Auer, Peter: Phonologie der Alltagssprache. Eine Untersuchung zur Standard/DialektVariation am Beispiel der Konstanzer Stadtsprache. - 1990. X, 358 S.

Behrens, Leila: Textanalyse und Sprachwandel. Historische Syntax des Ungarischen: ein empirisches Modell. - 1989. II, 480 S.

Bhatt, Christa: Die syntaktische Struktur der Nominalphrase im Deutschen. - 1990. VIII, $269 \mathrm{~S}$.

Birken-Silverman, Gabriele: Phonetische, morphosyntaktische und lexikalische Varianten in den palermitanischen Mundarten und im Sikuloalbanischen von Piana degli Albanesi. -1989 . XVII, $651 \mathrm{~S}$.

Deutsche Gegenwartssprache. Tendenzen und Perspektiven. Institut für deutsche Sprache - Jahrbuch 1989. Hrsg. von Gerhard Stickel. - 1990. 420 S.

Dialoganalyse I. Referate der 1. Arbeitstagung. Münster 1986. Hrsg. von Franz Hundsnurscher und Edda Weigand. - 1986. VIII, 432 S̆.

Dialoganalyse II. Referate der 2. Arbeitstagung. Bochum 1988. Hrsg. von Franz Hundsnurscher und Edda Weigand. - 1989. X, 478 S.

Dimensional adjectives. Grammatical structure and conceptual interpretation. Ed. by Manfred Bierwisch and Ewald Lang. - 1989. X, 523 S.

Einhauser, Eveline: Die Junggrammatiker. Ein Problem für die Sprachwissenschaftsgeschichtsschreibung. - 1990. 413 S.

English across cultures. Cultures across English. A reader in cross-cultural communication. Ed. by Ofelia Garcia and Ricardo Otheguy. - 1989. XXI, 492 S.

Fiehler, Reinhard: Kommunikation und Emotion. Theoretische und empirische Untersuchungen zur Rolle von Emotionen in der verbalen Interaktion. - 1990. VI, 335 S.

From gesture to language in hearing and deaf children. Ed. by Virginia Volterra and Carol J. Erting. - 1990. XV, $335 \mathrm{~S}$.

General and Amerindian ethnolinguistics in rememberence of Stanley Newman. Ed. by Mary Ritchie Key and Henry M. Hoenigswald. - 1989. XV, 499 S.

Graffe, Jürgen: Sich festlegen und verpflichten. Die Untertypen kommissiver Sprechakte und ihre sprachlichen Realisierungsformen. $-1990.321 \mathrm{~S}$.

Hagenbuchner, Albertine: Die Korrespondenz der Hethiter. Teil 1: Die Briefe unter ihren kulturellen; sprachlichen und thematischen Gesichtspunkten. - 1989. XXX, $175 \mathrm{~S}$.

-: Die Korrespondenz der Hethiter. Teil 2: Die Briefe mit Transkription, Übersetzung und Kommentar. - 1989. XLIV, 483 S.

Hellinger, Marlis: Kontrastive feministische Linguistik. Mechanismen sprachlicher Diskriminierung im Englischen und Deutschen. - 1990. 176 S. .

Herbermann; Clemens-Peter: Modi Referentiae. Studien zum sprachlichen Bezug zur Wirklichkeit. - 1988. 182 S.

Interaktion und Kommunikation mit dem Computer. Jahrestagung der Gesellschaft für linguistische Datenverarbeitung (GLDV) Ulm vom 8.-10. März 1989. Hrsg. von Brigitte Endres-Niggemeyer, Thomas Herrmann, Alfred Kobsa und Dietmar Rösner. - 1990, VIII, $175 \mathrm{~S}$.

Janicke, Karel: Toward non-essentialist sociolinguistics. - 1990. 136 S.

Kluge, Friedrich: Etymologisches Wörterbuch der deutschen Sprache. 22. Aufl. Unter Mithilfe von Max Bürgisser und Bernd Gregor völlig neu bearbeitet von Elmár Seebold. - 1989. LXV, 822S. 
Krifka, Manfred: Nominalreferenz und Zeitkonstitution. Zur Semantik von Massentermen, Pluraltermen und Aspektklassen. - 1990. 270 S.

Linguistic change and reconstruction methodology. Ed. by Philip Baldi. - 1990. XII, $752 \mathrm{~S}$.

Matzel, Klaus/Riecke, Jörg/Zipp, Gerhard: Spätmittelalterlicher deutscher Wortschatz aus Regensburger und mittelbairischen Quellen. - 1989. XL, 384 S.

Müller, Karin: „Schreibe, wie du sprichst!“" Eine Maxime im Spannungsfeld von Mündlichkeit und Schriftlichkeit. Eine historische und systematische Untersuchung. - 1990. XII, $512 \mathrm{~S}$.

Naumann, Carl Ludwig: Gesprochenes Deutsch und Orthographie. Linguistische und didaktische Studien zur Rolle der gesprochenen Sprache in System und Erwerb der Rechtschreibung. -1989 . 390 S.

Rechtsdiskurse. Untersuchungen zur Kommunikation in Gerichtsverfahren. Hrsg، von Ludger Hoffmann. - 1989. 326 S.

Reumuth, Wolfgang/Winkelmann, Otto: Praktische Grammatik der italienischen Sprache. 2., überarbeitete Auflage. - 1990. XII, 324 S.

Rice, Keren: A grammar of slave. - 1989. XLIV, 1370S.

Schmitz, Ulrich: Postmoderne Concierge: Die „Tagesschau“. Wortwelt und Weltbild der Fernsehnachrichten. - 1990. 383 S.

Vogt, Rüdiger: Gegenkulturelle Schreibweisen über Sexualität. Textstrukturen und soziale Praxis in Leserbriefen. - 1989. $309 \mathrm{~S}$.

Wode, Henning: Einführung in die Psycholinguistik. Theorien, Methoden, Ergebnisse. 1990. $400 \mathrm{~S}$.

Wörterbuch der vergleichenden Bezeichnungslehre. Europäisches Erbe und allgemeine Onomasiologie. Region Mittel-, Ost- und Südosteuropa. Band I: Ausgabe A. Lfg. 5/6. 1989. S. 195-354; Lfg. 7/8. 1989. S. 355-514.

Working with language. A multidisciplinary consideration of language use in work contexts. Ed. by Hywel Coleman. - 1989. XII, 617 S. 